\title{
On the Space of Functions with Growths Tempered by a Modulus of Continuity and Its Applications
}

\author{
Józef Banaś and Rafał Nalepa \\ Department of Mathematics, Rzeszów University of Technology, Powstańców Warszawy 8, 35-959 Rzeszów, Poland \\ Correspondence should be addressed to Józef Banaś; jbanas@prz.edu.pl
}

Received 7 February 2013; Accepted 20 March 2013

Academic Editor: Janusz Matkowski

Copyright ( 2013 J. Banaś and R. Nalepa. This is an open access article distributed under the Creative Commons Attribution License, which permits unrestricted use, distribution, and reproduction in any medium, provided the original work is properly cited.

\begin{abstract}
We are going to study the space of real functions defined on a bounded metric space and having growths tempered by a modulus of continuity. We prove also a sufficient condition for the relative compactness in the mentioned function space. Using that condition and the classical Schauder fixed point theorem, we show the existence theorem for some quadratic integral equations of Fredholm type in the space of functions satisfying the Hölder condition. An example illustrating the mentioned existence result is also included.
\end{abstract}

\section{Introduction}

The principal aim of the paper is to discuss the function space consisting of functions with growths tempered by a given modulus of continuity. Functions belonging to such a space have their moduli of continuity in some sense proportional to a given modulus of continuity. We assume here that the mentioned function space consists of real functions defined on a bounded metric space and, as we pointed out above, having growths tempered by a given modulus of continuity. Typical examples of function spaces of such a type are the space of functions satisfying the Lipschitz condition and the space of Hölder functions, that is, the space of functions satisfying the Hölder condition with a given exponent belonging to the interval $(0,1]$.

It is a surprising fact that those spaces, although they are frequently used in mathematical investigations, were not intensively studied and described in mathematical literature (cf. [1-4]). It is probably caused by the fact that the norm in the mentioned function space is not convenient for use in comparison with the norm in the classical space of real functions being continuous on a compact set. On the other hand, there are no known convenient sufficient conditions for relative compactness of bounded subsets of the function space in question.
It is also worthwhile mentioning that it is very difficult to encounter some applications of the space of functions with growths tempered by a modulus of continuity in the theory of operator equations (functional, differential, integral, etc.), (cf. $[5,6]$ ).

In this paper we are going to discuss in detail the above mentioned function space consisting of real functions having growths tempered by a given modulus of continuity. We describe a general form of such a space and we point out some important particular cases of spaces of the described type.

Apart from this, we indicate some essential properties of the considered function space. Namely, we show that such a space is not a closed subspace of the space of all real continuous functions with the supremum norm, with respect to this norm. The consequence of this property is the fact that the norm introduced in the space of functions with tempered growths is not equivalent to the classical supremum norm. This causes that it is not possible to use the supremum norm in all considerations conducted in the mentioned space of functions with tempered growths.

The main result proved in the paper is a sufficient condition for relative compactness of bounded subset of the discussed function space. We also point out several particular cases of that result, especially in the space of functions satisfying the Hölder condition. 
The last part of the paper contains an application of the obtained results in proving a theorem on the existence of a solution of a quadratic integral equation in the space of functions satisfying the Hölder condition. An example showing the applicability of that theorem to a concrete quadratic integral equation is also included.

\section{The Space of Functions with Tempered Moduli of Continuity}

In this section we discuss the space of real functions defined on a given bounded metric space and having the growths tempered by a given modulus of continuity. Moreover, we will also study some important particular cases of that space.

To this end denote by $\mathbb{R}$ the set of all real numbers and put $\mathbb{R}_{+}=[0, \infty)$. A function $\omega: \mathbb{R}_{+} \rightarrow \mathbb{R}_{+}$is said to be $a$ modulus of continuity if $\omega(0)=0, \omega(\varepsilon)>0$ for $\varepsilon>0$, and $\omega$ is nondecreasing on $\mathbb{R}_{+}$.

We will also assume (but not always) that the modulus of continuity $\omega=\omega(\varepsilon)$ is continuous at $\varepsilon=0$, that is, $\omega(\varepsilon) \rightarrow 0$ as $\varepsilon \rightarrow 0$.

In order to clarify the concept of the modulus of continuity, let us assume that $(X, d)$ is a given bounded metric space. Denote by $C(X)$ the space of all real functions defined and continuous on the metric space $X$. For $x \in C(X)$ and for an arbitrary fixed number $\varepsilon>0$ let us define the quantity $\nu(x, \varepsilon)$ by the formula

$$
v(x, \varepsilon)=\sup \{|x(u)-x(v)|: u, v \in X, d(u, v) \leqslant \varepsilon\} .
$$

The function $\varepsilon \rightarrow v(x, \varepsilon)$ is called the modulus of continuity of the function $x$. Observe that in order to define this concept it is sufficient to consider the space $B(X)$ consisting of real functions defined and bounded on $X$.

Observe that if $x \in B(X)$, then the modulus of continuity of $x$, that is, the function $\varepsilon \rightarrow v(x, \varepsilon)$, is the modulus of continuity in the above defined sense. Obviously, this modulus is continuous at $\varepsilon=0$ if and only if $x$ is uniformly continuous on $X$.

In what follows let us fix a modulus of continuity $\omega=\omega(\varepsilon)$ and assume, as before, that $(X, d)$ is a given bounded metric space. Denote by $C_{\omega}(X)$ the set of all real functions defined on $X$ such that their growths are tempered by the modulus of continuity $\omega$. More precisely, a function $x=x(u)$ belongs to the set $C_{\omega}(X)$ provided $x: X \rightarrow \mathbb{R}$ and there exists a constant $K_{x}>0$ such that

$$
|x(u)-x(v)| \leqslant K_{x} \omega(d(u, v))
$$

for all $u, v \in X$. In other words, we have that $x \in C_{\omega}(X)$ if and only if the quantity

$$
\sup \left\{\frac{|x(u)-x(v)|}{\omega(d(u, v))}: u, v \in X, u \neq v\right\}
$$

is finite.

It is easy to check that the set $C_{\omega}(X)$ forms a linear space over the field of real numbers $\mathbb{R}$. Obviously, $C_{\omega}(X)$ is a linear subspace of the space $C(X)$.
In the sequel, let us notice that if $x \in C_{\omega}(X)$, then in view of (2) there exists a constant $K_{x}>0$ such that

$$
\nu(x, \varepsilon) \leqslant K_{x} \omega(\varepsilon)
$$

for any $\varepsilon>0$. Obviously the converse implication is also true.

Thus, we can say equivalently that the set $C_{\omega}(X)$ consists of all real functions defined on $X$ such that the moduli of continuity of those functions are tempered by the given modulus of continuity $\omega=\omega(\varepsilon)$.

Now, fix arbitrarily an element $u_{0} \in X$. For an arbitrary function $x \in C_{\omega}(X)$ we define the quantity $\|x\|$ by the formula

$$
\|x\|=\left|x\left(u_{0}\right)\right|+\sup \left\{\frac{|x(u)-x(v)|}{\omega(d(u, v))}: u, v \in X, u \neq v\right\} .
$$

Notice that $\|x\|<\infty$ for any $x \in C_{\omega}(X)$. Moreover, it can be shown that $\|\cdot\|$ is a norm in the space $C_{\omega}(X)$; that is, $C_{\omega}(X)$ is a normed space with the norm defined by (5).

We show that the norm defined by (5) is complete. To this end, take a Cauchy sequence $\left(x_{n}\right)$ in the space $C_{\omega}(X)$. Fix arbitrarily a number $\varepsilon>0$ and denote $D=$ $\max \{1, \omega(\operatorname{diam} X)\}$, where diam $X$ denotes the diameter of the metric space $X$. Then, we can find a natural number $n_{0}$ such that for $n, m \geqslant n_{0}$, we have that $\left\|x_{n}-x_{m}\right\| \leqslant \varepsilon / 2 D$ or, by (5),

$$
\begin{gathered}
\left|x_{n}\left(u_{0}\right)-x_{m}\left(u_{0}\right)\right| \\
+\sup \left\{\frac{\left|\left[x_{n}(u)-x_{m}(u)\right]-\left[x_{n}(v)-x_{m}(v)\right]\right|}{\omega(d(u, v))}\right. \\
: u, v \in X, u \neq v\} \leqslant \frac{\varepsilon}{2 D} .
\end{gathered}
$$

Further, let us notice that from (6) we infer that

$$
\left|x_{n}\left(u_{0}\right)-x_{m}\left(u_{0}\right)\right| \leqslant \frac{\varepsilon}{2 D} \leqslant \frac{\varepsilon}{2} .
$$

This means that the real sequence $\left\{x_{n}\left(u_{0}\right)\right\}$ satisfies the Cauchy condition in $\mathbb{R}$ with natural metric. Hence we obtain that this sequence is convergent to a real number, say $x\left(u_{0}\right)$, that is, $\lim _{n \rightarrow \infty} x_{n}\left(u_{0}\right)=x\left(u_{0}\right)$.

Next, keeping in mind (6) again we deduce that

$$
\begin{gathered}
\sup \left\{\frac{\left|\left[x_{n}(u)-x_{m}(u)\right]-\left[x_{n}(v)-x_{m}(v)\right]\right|}{\omega(d(u, v))}\right. \\
: u, v \in X, u \neq v\} \leqslant \frac{\varepsilon}{2 D} .
\end{gathered}
$$

Putting in the above inequality $v=u_{0}$, we get that for each $u \in X, u \neq u_{0}$, the following estimate holds

$$
\frac{\left|\left[x_{n}(u)-x_{m}(u)\right]-\left[x_{n}\left(u_{0}\right)-x_{m}\left(u_{0}\right)\right]\right|}{\omega\left(d\left(u, u_{0}\right)\right)} \leqslant \frac{\varepsilon}{2 D} .
$$

This yields the inequality

$$
\left|\left[x_{n}(u)-x_{m}(u)\right]-\left[x_{n}\left(u_{0}\right)-x_{m}\left(u_{0}\right)\right]\right| \leqslant \frac{\varepsilon}{2 D} \omega\left(d\left(u, u_{0}\right)\right)
$$


which is valid for $u \in X$ and $m, n \in \mathbb{N}, n, m \geqslant n_{0}$ ( $\mathbb{N}$ denotes the set of natural numbers).

From that last inequality we get

$$
\left|x_{n}(u)-x_{m}(u)\right|-\left|x_{n}\left(u_{0}\right)-x_{m}\left(u_{0}\right)\right| \leqslant \frac{\varepsilon}{2 D} \omega\left(d\left(u, u_{0}\right)\right) \leqslant \frac{\varepsilon}{2} .
$$

Hence we obtain

$$
\left|x_{n}(u)-x_{m}(u)\right| \leqslant\left|x_{n}\left(u_{0}\right)-x_{m}\left(u_{0}\right)\right|+\frac{\varepsilon}{2}
$$

for an arbitrary $u \in X$ and $n, m \geqslant n_{0}$.

Linking the above inequality and (7), we deduce that

$$
\left|x_{n}(u)-x_{m}(u)\right| \leqslant \varepsilon
$$

for $u \in X$ and for $m, n \in \mathbb{N}, n, m \geqslant n_{0}$. But this fact means that $\left(x_{n}(u)\right)$ is a real Cauchy sequence for arbitrarily fixed $u \in$ $X$. Thus this sequence is convergent in $\mathbb{R}$. Denote its limit by $x(u)$, that is,

$$
x(u)=\lim _{n \rightarrow \infty} x_{n}(u) .
$$

Passing in (13) with $m \rightarrow \infty$, we obtain

$$
\left|x_{n}(u)-x(u)\right| \leqslant \varepsilon
$$

for any $u \in X$ and for $n \in \mathbb{N}, n \geqslant n_{0}$. This means that the sequence $\left(x_{n}\right)$ converges uniformly to the function $x$ on the metric space $X$.

Now, we show that the sequence $\left(x_{n}\right)$ converges to the function $x$ in the sense of norm (5). To this end we prove first that $x \in C_{\omega}(X)$. Indeed, taking into account the fact that $\left(x_{n}\right)$ is a Cauchy sequence in the space $C_{\omega}(X)$, we infer that $\left(x_{n}\right)$ is bounded in $C_{\omega}(X)$; that is, there exists a constant $M>0$ such that $\left\|x_{n}\right\| \leqslant M$ for $n=1,2, \ldots$. Hence, in view of (5) we have

$$
\sup \left\{\frac{\left|x_{n}(u)-x_{n}(v)\right|}{\omega(d(u, v))}: u, v \in X, u \neq v\right\} \leqslant M
$$

This implies that for arbitrarily fixed $u, v \in X, u \neq v$ and for $n \in \mathbb{N}$, we get

$$
\frac{\left|x_{n}(u)-x_{n}(v)\right|}{\omega(d(u, v))} \leqslant M
$$

and, consequently,

$$
\left|x_{n}(u)-x_{n}(v)\right| \leqslant M \omega(d(u, v))
$$

for all $u, v \in X$ and for $n \in \mathbb{N}$.
Now, fix arbitrarily $u, v \in X$. Then, passing in the above inequality with $n \rightarrow \infty$, we obtain

$$
|x(u)-x(v)| \leqslant M \omega(d(u, v))
$$

for all $u, v \in X$. But this means that $x \in C_{\omega}(X)$.

In what follows, using the fact that the sequence $\left(x_{n}\right)$ satisfies inequality (6), we derive that for arbitrary $u, v \in$ $X, u \neq v$ and for arbitrary $n, m \in \mathbb{N}, n, m \geqslant n_{0}$, the following inequality holds

$$
\frac{\left|\left[x_{n}(u)-x_{m}(u)\right]-\left[x_{n}(v)-x_{m}(v)\right]\right|}{\omega(d(u, v))} \leqslant \frac{\varepsilon}{2 D} .
$$

Fixing $u, v$ and passing with $m \rightarrow \infty$, we get

$$
\frac{\left|\left[x_{n}(u)-x(u)\right]-\left[x_{n}(v)-x(v)\right]\right|}{\omega(d(u, v))} \leqslant \frac{\varepsilon}{2 D} .
$$

Hence, in virtue of the arbitrariness of the choice of points $u, v \in X, u \neq v$, we have the estimate

$$
\begin{gathered}
\sup \left\{\frac{\left|\left[x_{n}(u)-x(u)\right]-\left[x_{n}(v)-x(v)\right]\right|}{\omega(d(u, v))}\right. \\
: u, v \in X, u \neq v\} \leqslant \frac{\varepsilon}{2 D}
\end{gathered}
$$

being valid for $n \geqslant n_{0}$.

In a similar way, keeping in mind inequality (6), we infer that

$$
\left|x_{n}\left(u_{0}\right)-x_{m}\left(u_{0}\right)\right| \leqslant \frac{\varepsilon}{2 D}
$$

for $n, m \in \mathbb{N}, n, m \geqslant n_{0}$. Hence, for $m \rightarrow \infty$, we obtain

$$
\left|x_{n}\left(u_{0}\right)-x\left(u_{0}\right)\right| \leqslant \frac{\varepsilon}{2 D} \text {. }
$$

Now, combining (22), (24), and (5), we obtain that

$$
\left\|x_{n}-x\right\| \leqslant \frac{\varepsilon}{D} \leqslant \varepsilon
$$

for all $n \in \mathbb{N}, n \geqslant n_{0}$. This means that the sequence $\left(x_{n}\right)$ is convergent in the space $C_{\omega}(X)$ to the function $x$. Thus, $C_{\omega}(X)$ is a Banach space.

Now, we provide a few examples.

Example 1. Take $\omega_{L}(\varepsilon)=\varepsilon$ for $\varepsilon \in \mathbb{R}_{+}$. Obviously $\omega_{L}$ is a modulus of continuity. Moreover, notice that $x \in C_{\omega_{L}}(X)$ if and only if there exists a constant $L_{x}>0$ such that

$$
|x(u)-x(v)| \leqslant L_{x} d(u, v)
$$

for $u, v \in X$. This means that the space $C_{\omega_{L}}(X)$ consists of functions $x: X \rightarrow \mathbb{R}$ satisfying the Lipschitz condition. The norm in the space $C_{\omega_{L}}(X)$ has the form

$$
\|x\|_{L}=\left|x\left(u_{0}\right)\right|+\sup \left\{\frac{|x(u)-x(v)|}{d(u, v)}: u, v \in X, u \neq v\right\} .
$$


Example 2. Let $\omega_{H}$ denote the modulus of continuity which corresponds to the Hölder condition. This means that $\omega_{H}(\varepsilon)=\varepsilon^{\alpha}$ for $\varepsilon \geqslant 0$, where $\alpha$ is a fixed number from the interval $(0,1]$.

Observe that $x \in C_{\omega_{H}}(X)$ if and only if there exists a constant $H_{x}>0$ such that

$$
|x(u)-x(v)| \leqslant H_{x}(d(u, v))^{\alpha}
$$

for all $u, v \in X$. In other words, the function $x$ satisfies the Hölder condition with a constant $H_{x}$ and with an exponent $\alpha$. Notice that the norm in the space $C_{\omega_{H}}(X)$ has the from

$$
\|x\|_{H}=\left|x\left(u_{0}\right)\right|+\sup \left\{\frac{|x(u)-x(v)|}{(d(u, v))^{\alpha}}: u, v \in X, u \neq v\right\} .
$$

Example 3. Now, let us take into account the modulus of continuity $\omega$ having the form

$$
\omega(\varepsilon)= \begin{cases}0 & \text { for } \varepsilon=0 \\ 1 & \text { for } \varepsilon>0\end{cases}
$$

Note that $x \in C_{\omega}(X)$ if and only if there exists a constant $K_{x}>$ 0 such that $|x(u)-x(v)| \leqslant K_{x}$ for $u, v \in X$. Obviously, this condition is equivalent to the boundedness of the function $x$. Indeed, fix $u_{0} \in X$. Then, for any $u \in X$ we have

$$
|x(u)|-\left|x\left(u_{0}\right)\right| \leqslant\left|x(u)-x\left(u_{0}\right)\right| \leqslant K_{x} .
$$

This yields

$$
|x(u)| \leqslant\left|x\left(u_{0}\right)\right|+K_{x}<\infty
$$

for $u \in X$. The converse implication is also obvious. Thus we can write that $C_{\omega}(X)=B(X)$; that is, $C_{\omega}(X)$ contains all functions defined and bounded on $X$. Further, observe that the norm in the space $C_{\omega}(X)$ has now the form

$$
\|x\|=\left|x\left(u_{0}\right)\right|+\sup _{u, v \in X}|x(u)-x(v)| .
$$

It is easy to show that this norm is equivalent to the classical supremum norm $\|\cdot\|_{\infty}$ in the space $B(X)$. Indeed, we have

$$
\|x\|_{\infty} \leqslant\|x\| \leqslant 3\|x\|_{\infty}
$$

for any function $x \in B(X)$, where $\|x\|_{\infty}=\sup _{u \in X}|x(u)|$.

The examples of the functions $x(u)=u-1$ and $x(u)=u$ in the space $B([0,2])$ with $u_{0}=0$ show that the equality signs in (34) are attained.

\section{Some Properties of the Space of Hölder Functions}

In order to simplify the considerations in this section, we restrict ourselves to spaces of real functions defined on a fixed interval $[a, b]$. In this way we will consider the space $C[a, b]$ consisting of functions $x:[a, b] \rightarrow \mathbb{R}$, which are continuous on $[a, b]$ and equipped with the norm $\|x\|_{\infty}=\sup \{|x(t)|: t \in$ $[a, b]\}$.

Further, if $\alpha(0<\alpha \leqslant 1)$ is a fixed number, then the symbol $H_{\alpha}[a, b]$ will denote the space $C_{\omega_{H}}([a, b])$ (see Example 2) with $\omega_{H}=\varepsilon^{\alpha}$; that is, $H_{\alpha}[a, b]$ is the collection of all real functions $x$ defined on $[a, b]$ and satisfying the Hölder condition. More precisely, $x \in H_{\alpha}[a, b]$ if there exists a constant $H_{x}^{\alpha}$ such that

$$
|x(t)-x(s)| \leqslant H_{x}^{\alpha}|t-s|^{\alpha}
$$

for all $t, s \in[a, b]$.

Observe that $H_{\alpha}[a, b]$ forms a linear subspace of the linear space $C[a, b]$.

In what follows, for $x \in H_{\alpha}[a, b]$, the symbol $H_{x}^{\alpha}$ will denote the least possible constant for which inequality (35) is satisfied. In other words, we can write

$$
H_{x}^{\alpha}=\sup \left\{\frac{|x(t)-x(s)|}{|t-s|^{\alpha}}: t, s \in[a, b], t \neq s\right\}
$$

Notice that in the case $\alpha=1$, the linear space $H_{1}[a, b]$ coincides with the space of real functions defined on $[a, b]$ and satisfying the Lipschitz condition, that is,

$$
|x(t)-x(s)| \leqslant L_{x}|t-s|
$$

for some constant $L_{x}>0$ and for all $t, s \in[a, b]$. This space will be denoted by the symbol Lip $[a, b]$.

Our principal aim is to show that the spaces $H_{\alpha}[a, b]$ (for $\alpha \in(0,1])$ do not form closed subspaces of the space $C[a, b]$ with respect to the norm $\|\cdot\|_{\infty}$. In order to prove this fact, observe that the space $H_{\alpha}[a, b]$ can be normed in a natural way (cf. Example 2) if for $x \in H_{\alpha}[a, b]$ we put

$$
\|x\|_{\alpha}=|x(a)|+\sup \left\{\frac{|x(t)-x(s)|}{|t-s|^{\alpha}}: t, s \in[a, b], t \neq s\right\} .
$$

Observe that in view of (36), the above definition can be written in the from

$$
\|x\|_{\alpha}=|x(a)|+H_{x}^{\alpha} .
$$

Obviously, the space $H_{\alpha}[a, b]$ with the norm (38) is the Banach space (cf. Section 2). 
Further on let us notice that for an arbitrarily fixed $x \in$ $H_{\alpha}[a, b]$ and for an arbitrary $t \in[a, b]$ we obtain

$$
\begin{aligned}
& |x(t)| \\
& \leqslant|x(t)-x(a)|+|x(a)| \\
& \leqslant|x(a)|+\sup \{|x(t)-x(a)|: t \in[a, b]\} \\
& \leqslant|x(a)|+\sup \{|x(t)-x(s)|: t, s \in[a, b]\} \\
& =|x(a)|+\sup \left\{\frac{|x(t)-x(s)|}{|t-s|^{\alpha}|t-s|^{\alpha}}\right. \\
& \quad: t, s \in[a, b], \quad t \neq s\} \\
& \quad \times(b-a)^{\alpha} \quad\left\{\frac{|x(t)-x(s)|}{|t-s|^{\alpha}}: t, s \in[a, b], t \neq s\right\} \\
& \leqslant \max \left\{1,(b-a)^{\alpha}\right\}\left\{|x(a)|+\sup \left\{\frac{|x(t)-x(s)|}{|t-s|^{\alpha}}\right.\right. \\
& \quad: t, s \in[a, b], t \neq s\}\} .
\end{aligned}
$$

Hence we get

$$
\|x\|_{\infty} \leqslant \max \left\{1,(b-a)^{\alpha}\right\}\|x\|_{\alpha} .
$$

The above inequality means that the norm $\|\cdot\|_{\infty}$ is dominated by the norm $\|\cdot\|_{\alpha}$.

If the space $H_{\alpha}[a, b]$ would be a closed subspace of $C[a, b]$ with respect to the norm $\|\cdot\|_{\infty}$, then this would mean that $H_{\alpha}[a, b]$ (as the linear space) is a Banach space with respect to the norm $\|\cdot\|_{\infty}$. In other words, the space $H_{\alpha}[a, b]$ would be a Banach space both with respect to the norm $\|\cdot\|_{\infty}$ and with respect to the norm $\|\cdot\|_{\alpha}$. Hence, keeping in mind (41) and taking into account the theorem on the equivalence of dominated norms [4], we would infer that the norms $\|\cdot\|_{\infty}$ and $\|\cdot\|_{\alpha}$ are equivalent in the space $H_{\alpha}[a, b]$.

We show that such a conclusion is not true.

To this end fix a number $\alpha \in(0,1]$ and consider the sequence $\left(x_{n}\right)$ of real functions defined on the interval $[0,1]$ by the formula

$$
x_{n}(t)= \begin{cases}n^{\alpha} t^{\alpha} & \text { for } t \in\left[0, \frac{1}{n}\right), \\ 1 & \text { for } t \in\left[\frac{1}{n}, 1\right]\end{cases}
$$

for $n=1,2, \ldots$ Observe that $\left(x_{n}\right) \subset C[0,1]$ and $\left\|x_{n}\right\|_{\infty}=1$ for $n=1,2, \ldots$ On the other hand, we have

$$
\frac{x_{n}(t)-x_{n}(0)}{t^{\alpha}}=\frac{n^{\alpha} t^{\alpha}}{t^{\alpha}}=n^{\alpha}, \quad \text { for } t \in(0,1] \text {. }
$$

This implies that $\left\|x_{n}\right\|_{\alpha}=n^{\alpha}$, for $n=1,2, \ldots$ Hence we get

$$
\frac{\left\|x_{n}\right\|_{\infty}}{\left\|x_{n}\right\|_{\alpha}}=\frac{1}{n^{\alpha}}
$$

which shows that $\left\|x_{n}\right\|_{\infty} /\left\|x_{n}\right\|_{\alpha} \rightarrow 0$ as $n \rightarrow \infty$. Thus the norms $\|\cdot\|_{\infty}$ and $\|\cdot\|_{\alpha}$ are not equivalent in the space $H_{\alpha}[0,1]$. Hence we infer that $H_{\alpha}[0,1]$ is not closed subspace of the space $C[0,1]$ with respect to the norm $\|\cdot\|_{\infty}$. Obviously the same assertion is true if we replace the interval $[0,1]$ by an arbitrary interval $[a, b]$.

In what follows, let us observe that for $0<\alpha<\gamma \leqslant 1$ the following inclusions hold:

$$
H_{\gamma}[a, b] \subset H_{\alpha}[a, b] \subset C[a, b] .
$$

Particularly, taking into account that for $\gamma=1$, we have

$$
H_{1}[a, b]=\operatorname{Lip}[a, b],
$$

which yields

$$
\operatorname{Lip}[a, b] \subset H_{\gamma}[a, b] \subset H_{\alpha}[a, b] \subset C[a, b]
$$

for $0<\alpha<\gamma<1$.

Indeed, if $x \in H_{\gamma}[a, b]$, we obtain

$$
\begin{aligned}
|x(t)-x(s)| & \leqslant H_{x}^{\gamma}|t-s|^{\gamma}=H_{x}^{\gamma}|t-s|^{\alpha} \cdot|t-s|^{\gamma-\alpha} \\
& \leqslant(b-a)^{\gamma-\alpha} H_{x}^{\gamma}|t-s|^{\alpha} .
\end{aligned}
$$

This shows that $x \in H_{\alpha}[a, b]$ and hence we infer that inclusions (47) hold.

Further, for $x \in H_{\gamma}[a, b]$ and for arbitrarily fixed $t, s \in$ $[a, b], t \neq s$, we derive

$$
\begin{aligned}
\frac{|x(t)-x(s)|}{|t-s|^{\alpha}} & =\frac{|x(t)-x(s)|}{|t-s|^{\gamma}}|t-s|^{\gamma-\alpha} \\
& \leqslant \frac{|x(t)-x(s)|}{|t-s|^{\gamma}}(b-a)^{\gamma-\alpha} .
\end{aligned}
$$

Hence we get

$$
\begin{gathered}
\|x\|_{\alpha}=|x(a)|+\sup \left\{\frac{|x(t)-x(s)|}{|t-s|^{\alpha}}: t, s \in[a, b], t \neq s\right\} \\
\leqslant|x(a)|+\sup \left\{\frac{|x(t)-x(s)|}{|t-s|^{\gamma}}\right. \\
: t, s \in[a, b], t \neq s\}(b-a)^{\gamma-\alpha} \\
\leqslant \max \left\{1,(b-a)^{\gamma-\alpha}\right\}\|x\|_{\gamma}
\end{gathered}
$$

for $x \in H_{\gamma}[a, b]$. Thus the norm $\|\cdot\|_{\alpha}$ is dominated by the $\operatorname{norm}\|\cdot\|_{\gamma}^{\gamma}$.

We show that the inverse assertion is not true. To this end, take the function sequence $\left(x_{n}\right) \subset H_{\gamma}[0,1]$, where $x_{n}$ is defined by the formula

$$
x_{n}(t)= \begin{cases}n^{\gamma} t^{\gamma} & \text { if } t \in\left[0, \frac{1}{n}\right), \\ 1 & \text { if } t \in\left[\frac{1}{n}, 1\right]\end{cases}
$$


for $n=1,2, \ldots$ It is easily seen that

$$
\begin{aligned}
\left\|x_{n}\right\|_{\gamma}=\left|x_{n}(0)\right|+\sup \left\{\frac{\left|x_{n}(t)-x_{n}(s)\right|}{|t-s|^{\gamma}}\right. \\
: t, s \in[0,1], t \neq s\} \\
=\sup \left\{\frac{n^{\gamma} t^{\gamma}}{t^{\gamma}}: t \in\left(0, \frac{1}{n}\right]\right\}=n^{\gamma}
\end{aligned}
$$

for $=1,2, \ldots$ On the other hand, we have

$$
\begin{aligned}
\left\|x_{n}\right\|_{\alpha} & =\sup \left\{\frac{n^{\gamma} t^{\gamma}}{t^{\alpha}}: t \in\left(0, \frac{1}{n}\right]\right\} \\
& =\sup \left\{n^{\gamma} t^{\gamma-\alpha}: t \in\left[0, \frac{1}{n}\right]\right\}=n^{\alpha}
\end{aligned}
$$

for $n=1,2, \ldots$. Hence we get

$$
\frac{\left\|x_{n}\right\|_{\alpha}}{\left\|x_{n}\right\|_{\gamma}}=\frac{1}{n^{\gamma-\alpha}}
$$

which yields that $\left\|x_{n}\right\|_{\alpha} /\left\|x_{n}\right\|_{\gamma} \rightarrow 0$ as $n \rightarrow \infty$. This shows that the norm $\|\cdot\|_{\gamma}$ is not dominated by the norm $\|\cdot\|_{\alpha}$. Thus these norms are not equivalent.

\section{A Sufficient Condition for Relative Compactness in the Space $C_{\omega}(X)$}

This section is devoted to present a criterion for relative compactness in the space $C_{\omega}(X)$ of functions with growths tempered by a given modulus of continuity $\omega=\omega(\varepsilon)$. More precisely, we will discuss a sufficient condition for relative compactness in $C_{\omega}(X)$.

It is worthwhile mentioning that as far as we know, there are no known conditions of such a type (e.g., cf. [1-4]).

Let us recall that the complete description of the space $C_{\omega}(X)$ was given in Section 2.

Theorem 4. Assume that $\omega=\omega(\varepsilon)$ is a given modulus of continuity such that $\omega(\varepsilon) \rightarrow 0$ as $\varepsilon \rightarrow 0$, and assume that $X$ is a compact metric space. Let $A$ be a bounded subset of the space $C_{\omega}(X)$ such that functions belonging to $A$ are equicontinuous with respect to the modulus of continuity $\omega$; that is, the following condition is satisfied:

$$
\begin{gathered}
\forall_{\varepsilon>0} \exists_{\delta>0} \forall_{x \in A} \forall_{\substack{u, v \in X \\
u \neq v}}\left[d(u, v) \leqslant \delta \Longrightarrow \frac{|x(u)-x(v)|}{\omega(d(u, v))}\right. \\
\leqslant \varepsilon] .
\end{gathered}
$$

Then the set $A$ is relatively compact in the space $C_{\omega}(X)$.
Proof. At first, let us observe that since $A$ is bounded, then there exists a constant $M>0$ such that for an arbitrary $x \in A$ the following inequality holds:

$$
\begin{aligned}
\|x\|=\left|x\left(u_{0}\right)\right|+\sup \left\{\frac{|x(u)-x(v)|}{\omega(d(u, v))}\right. \\
\quad: u, v \in X, u \neq v\} \leqslant M,
\end{aligned}
$$

where $u_{0}$ is a fixed element of the metric space $X$ (cf. (5)).

Particularly, we conclude that

$$
\left|x\left(u_{0}\right)\right| \leqslant M
$$

for an arbitrary $x \in A$. Moreover, on the basis of (56) we infer that for any $x \in A$ and for arbitrary $u, v \in X, u \neq v$, the following inequality holds:

$$
\frac{|x(u)-x(v)|}{\omega(d(u, v))} \leqslant M .
$$

The above inequality yields

$$
|x(u)-x(v)| \leqslant M \omega(d(u, v))
$$

for an arbitrary function $x \in A$ and for all $u, v \in X$.

Since $\lim _{\varepsilon \rightarrow 0} \omega(\varepsilon)=0$, from (59) we deduce that all functions belonging to the set $A$ are equicontinuous on the set $X$. Apart from that, from (59) we have that for an arbitrary $x \in A$ the inequality

$$
|x(u)-x(v)| \leqslant M \omega(\operatorname{diam} X)
$$

holds for all $u, v \in X$. Putting in the above inequality $v=u_{0}$, we infer that for arbitrary $x \in A$ and $u \in X$ the following estimate is satisfied:

$$
|x(u)|-\left|x\left(u_{0}\right)\right| \leqslant\left|x(u)-x\left(u_{0}\right)\right| \leqslant M \omega(\operatorname{diam} X) .
$$

This implies that

$$
|x(u)| \leqslant\left|x\left(u_{0}\right)\right|+M \omega(\operatorname{diam} X)
$$

for $x \in A$ and $u \in X$. This means that functions from the set $A$ are equibounded.

Now, let us consider an arbitrary sequence $\left(x_{n}\right)$ in the set $A$. Taking into account the above established facts, we infer that functions of the sequence $\left(x_{n}\right)$ are equibounded and equicontinuous on the set $X$. Hence, in view of AscoliArzéla criterion we derive that there exists a subsequence of the sequence $\left(x_{n}\right)$ which is uniformly convergent on the set $X$ to a function $x=x(u)$. To avoid complicated notation, we will denote the mentioned subsequence of the sequence $\left(x_{n}\right)$ by the same symbol $\left(x_{n}\right)$. Observe that the function $x=x(u)$ is continuous on the set $X$. 
In what follows, we show that $x \in C_{\omega}(X)$. To this end observe that from the fact that functions belonging to the sequence $\left(x_{n}\right)$ satisfy inequality (59), we have that

$$
\left|x_{n}(u)-x_{n}(v)\right| \leqslant M \omega(d(u, v))
$$

for an arbitrary $n \in \mathbb{N}$ and for $u, v \in X$. Since $\lim _{n \rightarrow \infty} x_{n}(z)=$ $x(z)$ for an arbitrary element $z \in X$, thus keeping in mind the continuity of the absolute value and other standard facts from mathematical analysis, we deduce from the last inequality that

$$
|x(u)-x(v)| \leqslant M \omega(d(u, v))
$$

for arbitrary $u, v \in X$.

Similarly, on the basis of (57), we have

$$
\left|x_{n}\left(u_{0}\right)\right| \leqslant M
$$

for $n=1,2 \ldots$ Hence we obtain that

$$
\left|x\left(u_{0}\right)\right| \leqslant M \text {. }
$$

Combining (64) and (66) we derive the following estimate:

$$
\left|x\left(u_{0}\right)\right|+\frac{|x(u)-x(v)|}{\omega(d(u, v))} \leqslant 2 M
$$

for arbitrary $u, v \in X, u \neq v$. This implies that

$$
\left|x\left(u_{0}\right)\right|+\sup \left\{\frac{|x(u)-x(v)|}{\omega(d(u, v))}: u, v \in X, u \neq v\right\} \leqslant 2 M
$$

which shows that $x \in C_{\omega}(X)$.

Further on we show that the sequence $\left(x_{n}\right)$ is convergent to the function $x$ in the sense of the norm of the space $C_{\omega}(X)$.

To this end for convenience, let us denote

$$
\begin{gathered}
X_{0}^{2}=\{(u, v) \in X \times X: u \neq v\}, \\
X_{\delta}^{2}=\left\{(u, v) \in X_{0}^{2}: d(u, v) \leq \delta\right\}, \\
\widetilde{X_{\delta}^{2}}=X_{0}^{2} \backslash X_{\delta}^{2},
\end{gathered}
$$

where $\delta>0$ is a fixed number

Obviously we have that $X_{0}^{2}=X_{\delta}^{2} \cup \widetilde{X_{\delta}^{2}}$ and the sets $X_{\delta}^{2}, \widetilde{X_{\delta}^{2}}$ are disjoint.

Further, for an arbitrarily fixed natural number $n$, we obtain

$$
\begin{aligned}
\left\|x_{n}-x\right\|= & \left|x_{n}\left(u_{0}\right)-x\left(u_{0}\right)\right| \\
& +\sup _{(u, v) \in X_{0}^{2}}\left\{\frac{\left|x_{n}(u)-x(u)-x_{n}(v)+x(v)\right|}{\omega(d(u, v))}\right\} \\
= & \left|x_{n}\left(u_{0}\right)-x\left(u_{0}\right)\right| \\
& +\max \left\{\sup _{(u, v) \in \widetilde{X_{\delta}^{2}}} \frac{\left|x_{n}(u)-x(u)-x_{n}(v)+x(v)\right|}{\omega(d(u, v))},\right. \\
& \left.\sup _{(u, v) \in X_{\delta}^{2}} \frac{\left|x_{n}(u)-x(u)-x_{n}(v)+x(v)\right|}{\omega(d(u, v))}\right\} .
\end{aligned}
$$

Next, fix arbitrarily a number $\varepsilon>0$. Let $\delta>0$ denote a number corresponding to $\varepsilon / 4$ according to the assumption about equicontinuity of functions from the set $A$ with respect to the modulus of continuity $\omega=\omega(\varepsilon)$.

Now, denote by $\beta(\varepsilon)$ the number defined as follows:

$$
\beta(\varepsilon)=\min \left\{\frac{\varepsilon}{2}, \frac{\varepsilon \omega(\delta)}{4}\right\}
$$

Keeping in mind the fact that functions of the sequence $\left(x_{n}\right)$ are uniformly convergent on the set $X$ to a function $x$, let us choose a natural number $n_{0}$ such that

$$
\left|x_{n}(w)-x(w)\right| \leq \beta(\varepsilon)
$$

for all $n \geqslant n_{0}$ and for any $w \in X$.

Further, let us observe that $d(u, v)>\delta$ for $(u, v) \in \widetilde{X_{\delta}^{2}}$. Hence we infer that $\omega(d(u, v)) \geqslant \omega(\delta)$. Thus, for an arbitrary $n \geqslant n_{0}$, in view of (71) and (72) we get

$$
\begin{aligned}
& \sup _{(u, v) \in \widetilde{X_{\delta}^{2}}}\left\{\frac{\left|x_{n}(u)-x(u)-x_{n}(v)+x(v)\right|}{\omega(d(u, v))}\right\} \\
& \leqslant \sup _{(u, v) \in \widetilde{X_{\delta}^{2}}}\left\{\frac{\left|x_{n}(u)-x(u)\right|+\left|x_{n}(v)-x(v)\right|}{\omega(d(u, v))}\right\} \\
& \leqslant \sup _{(u, v) \in \widetilde{X_{\delta}^{2}}}\left\{\frac{\left|x_{n}(u)-x(u)\right|+\left|x_{n}(v)-x(v)\right|}{\omega(\delta)}\right\} \\
& \leqslant 2 \frac{\beta(\varepsilon)}{\omega(\delta)} \leq \frac{\varepsilon}{2} .
\end{aligned}
$$

Next, taking into account the fact that the functions of the sequence $\left(x_{n}\right)$ belong to the set $A$, in virtue of the choice of the number $\delta$ to the number $\varepsilon / 4$, we infer that for an arbitrary pair $(u, v) \in X_{\delta}^{2}$ and for an arbitrary natural number $n$ the following inequality is satisfied:

$$
\frac{\left|x_{n}(u)-x_{n}(v)\right|}{\omega(d(u, v))} \leqslant \frac{\varepsilon}{4}
$$

Hence we obtain

$$
\left|x_{n}(u)-x_{n}(v)\right| \leqslant \frac{\varepsilon}{4} \omega(d(u, v))
$$

for $(u, v) \in X$ such that $d(u, v) \leqslant \delta$. 
Letting in the above inequality with $n \rightarrow \infty$, we get

$$
|x(u)-x(v)| \leqslant \frac{\varepsilon}{4} \omega(d(u, v))
$$

for $(u, v) \in X$ such that $d(u, v) \leqslant \delta$. Consequently, we obtain

$$
\frac{|x(u)-x(v)|}{\omega(d(u, v))} \leqslant \frac{\varepsilon}{4}
$$

for arbitrary $(u, v) \in X_{\delta}^{2}$.

Now, in view of (74) and (77), we derive the following estimates:

$$
\begin{aligned}
& \sup _{(u, v) \in X_{\delta}^{2}}\left\{\frac{\left|x_{n}(u)-x(u)-x_{n}(v)+x(v)\right|}{\omega(d(u, v))}\right\} \\
\leqslant & \sup _{(u, v) \in X_{\delta}^{2}}\left\{\frac{\left|x_{n}(u)-x_{n}(v)\right|+|x(u)-x(v)|}{\omega(d(u, v))}\right\} \leqslant \frac{\varepsilon}{2} .
\end{aligned}
$$

Combining (70) with inequalities (72), (73), and (78), we obtain that $\left\|x_{n}-x\right\| \leqslant \varepsilon$ for $n \in \mathbb{N}, n \geqslant n_{0}$. This means that the sequence $\left(x_{n}\right)$ is convergent to the function $x$ with respect to the norm of the space $C_{\omega}(X)$. Finally, we conclude that the set $A$ is relatively compact in the space $C_{\omega}(X)$ and the proof is complete.

Now, based on Theorem 4, we prove a manageable and handy sufficient condition for relative compactness in the space $C_{\omega}(X)$.

Theorem 5. Assume that $\omega_{1}, \omega_{2}$ are moduli of continuity being continuous at zero and such that $\omega_{2}(\varepsilon)=o\left(\omega_{1}(\varepsilon)\right)$ as $\varepsilon \rightarrow 0$, that is,

$$
\lim _{\varepsilon \rightarrow 0} \frac{\omega_{2}(\varepsilon)}{\omega_{1}(\varepsilon)}=0
$$

Further, assume that $(X, d)$ is a compact metric space. Then, if $A$ is a bounded subset of the space $C_{\omega_{2}}(X)$ then $A$ is relatively compact in the space $C_{\omega_{1}}(X)$.

Proof. Since $A$ is bounded in the space $C_{\omega_{2}}(X)$, then there exists a constant $M>0$ such that $\|x\| \leqslant M$ for any $x \in A$, or equivalently

$$
\left|x\left(u_{0}\right)\right|+\sup \left\{\frac{|x(u)-x(v)|}{\omega_{2}(d(u, v))}: u, v \in X, u \neq v\right\} \leqslant M .
$$

Hence we infer that

$$
\frac{|x(u)-x(v)|}{\omega_{2}(d(u, v))} \leqslant M
$$

for all $u, v \in X, u \neq v$.

Further, fix an arbitrary number $P>0$. According to our assumptions there exists $\delta_{0}>0$ such that $\omega_{2}(t) / \omega_{1}(t) \leqslant P$ for $t \in\left(0, \delta_{0}\right]$. This yields that

$$
\omega_{2}(t) \leqslant P \omega_{1}(t)
$$

for $t \in\left[0, \delta_{0}\right]$.

Linking (81) and (82), we deduce that

$$
|x(u)-x(v)| \leqslant P M \omega_{1}(d(u, v))
$$

for all $u, v \in X$ such that $d(u, v) \leqslant \delta_{0}$.

Now, take arbitrary $u, v \in X$ such that $d(u, v) \geqslant \delta_{0}$. Then we get

$$
\begin{aligned}
\frac{|x(u)-x(v)|}{\omega_{1}(d(u, v))} & \leqslant \frac{|x(u)-x(v)|}{\omega_{1}\left(\delta_{0}\right)} \\
& =\frac{|x(u)-x(v)|}{\omega_{2}(d(u, v))} \cdot \frac{\omega_{2}(d(u, v))}{\omega_{1}\left(\delta_{0}\right)} .
\end{aligned}
$$

Hence, in view of (81), we obtain

$$
\frac{|x(u)-x(v)|}{\omega_{1}(d(u, v))} \leqslant M \frac{\omega_{2}(\operatorname{diam} X)}{\omega_{1}\left(\delta_{0}\right)}
$$

for all $u, v \in X$ such that $d(u, v) \geqslant \delta_{0}$.

Finally, joining (81), (82), and (85), we deduce that the set $A$ is bounded in the space $C_{\omega_{1}}(X)$.

In what follows, let us fix arbitrarily a number $\varepsilon_{1}>0$. Keeping in mind the assumption requiring that $\omega_{2}(t)=$ $o\left(\omega_{1}(t)\right)$ as $t \rightarrow 0$, we can find $\delta>0$ such that

$$
\frac{\omega_{2}(t)}{\omega_{1}(t)} \leqslant \varepsilon_{1}
$$

provided $0<t \leqslant \delta$.

Further, fix an arbitrary number $\varepsilon>0$. Then, according to (86), for the number $\varepsilon_{1}=\varepsilon / M$ let us choose $\delta>0$. Then, for $u, v \in X$ such that $u \neq v$ and $d(u, v) \leqslant \delta$ we get

$$
\frac{\omega_{2}(d(u, v))}{\omega_{1}(d(u, v))} \leqslant \frac{\varepsilon}{M}
$$

Hence we obtain

$$
\omega_{2}(d(u, v)) \leqslant \frac{\varepsilon}{M} \omega_{1}(d(u, v))
$$

for $u, v \in X, u \neq v$ and $d(u, v) \leqslant \delta$. 
Next, taking $u, v \in X$ such that $u \neq v, d(u, v) \leqslant \delta$ and keeping in mind (81), we derive the following estimate:

$$
|x(u)-x(v)| \leqslant M \omega_{2}(d(u, v)) \leqslant \varepsilon \omega_{1}(d(u, v)) .
$$

Hence we have

$$
\frac{|x(u)-x(v)|}{\omega_{1}(d(u, v))} \leqslant \varepsilon
$$

for $u, v \in X$ such that $u \neq v$ and $d(u, v) \leqslant \delta$.

Finally, in view of Theorem 4 we conclude that the set $A$ is relatively compact in the space $C_{\omega_{1}}(X)$. This completes the proof.

Example 6. In order to illustrate the applicability of Theorem 5, let us consider two moduli of continuity of Hölder type having the form $\omega_{1}(\varepsilon)=\varepsilon^{\alpha}, \omega_{2}(\varepsilon)=\varepsilon^{\beta}$, where $0<\alpha<\beta \leqslant 1$ (cf. Section 3). Then we have

$$
\lim _{\varepsilon \rightarrow 0} \frac{\omega_{2}(\varepsilon)}{\omega_{1}(\varepsilon)}=\lim _{\varepsilon \rightarrow 0} \varepsilon^{\beta-\alpha}=0 .
$$

This shows that the moduli of continuity $\omega_{1}(\varepsilon)$ and $\omega_{2}(\varepsilon)$ satisfy the conditions of Theorem 5 .

Thus, if we assume that a set $A$ is bounded in the space $C_{\omega_{2}}([a, b])$ or $C_{\omega_{2}}(X)$, then $A$ is relatively compact in the space $C_{\omega_{1}}([a, b])$ or $C_{\omega_{1}}(X)$, respectively, provided $(X, d)$ is a compact metric space.

In other words, if there exist numbers $\alpha, \beta$ with $0<\alpha<1$ and $\alpha<\beta \leqslant 1$ such that $A$ is a bounded set in the Hölder space with the exponent $\beta$, that is, there exists a constant $M>$ 0 such that

$$
|x(u)-x(v)| \leqslant M(d(u, v))^{\beta}
$$

for any $x \in A$ and for all $u, v \in X$, then the set $A$ is relatively compact in the Hölder space with the exponent $\alpha$.

\section{Application to a Quadratic Integral Equation}

In this final section we are going to present an application of the results obtained in previous sections to derive an existence result for a quadratic integral equation of Fredholm type. Equations of such a type occur naturally in connection with some problems investigated in the theories of radiative transfer, neutron transport, and the kinetic theory of gases [7-11]. Those integral equations are known as Chandrasekhar quadratic integral equations (cf. [10]).

Let us pay attention to some important facts connected with our considerations.

First of all, notice that the study of the existence of solutions of functional, differential, and integral equations is very complicated in the Lipschitz and Hölder function spaces which were considered in Section 3. To justify such an opinion, let us recall a result due to Matkowski [6] asserting that the so-called superposition operator generated by the function $f=f(t, x)$ maps the space $\operatorname{Lip}[a, b]$ into itself and is Lipschitzian (with respect to the norm in the space $\operatorname{Lip}[a, b]$ described in Example 1) if and only if the function $f$ is linear; that is, there exist functions $p, q \in \operatorname{Lip}[a, b]$ such that

$$
f(t, x)=p(t)+q(t) x
$$

for all $t \in[a, b]$ and $x \in \mathbb{R}$. Other results in this direction can be found in [5], for instance.

This shows that the investigations on the existence of solutions of operator equations in the space $C_{\omega}(X)$ consisting of functions with growths tempered by a modulus of continuity are rather a delicate matter.

As we indicate above, the object of the study conducted in this section is the quadratic integral equation of Fredholm type having the form

$$
x(t)=p(t)+x(t) \int_{a}^{b} k(t, \tau) x(\tau) d \tau,
$$

where $t \in[a, b]$.

In our further considerations we will assume that $\beta$ is a fixed number in the interval $(0,1]$.

Now, we formulate the assumptions under which we will study (94).

(i) The function $p=p(t)$ belongs to the Hölder space $H_{\beta}[a, b]$.

(ii) $k:[a, b] \times[a, b] \rightarrow \mathbb{R}$ is a continuous function such that it satisfies the Hölder condition with the exponent $\beta$ with respect to the first variable; that is, there exists a constant $k_{\beta}>0$ such that

$$
|k(t, \tau)-k(s, \tau)| \leqslant k_{\beta}|t-s|^{\beta}
$$

for all $t, s, \tau \in[a, b]$.

In what follows, based on the above assumptions, we can define the finite constant $K$ by putting

$$
K=\sup \left\{\int_{a}^{b}|k(t, \tau)| d \tau: t \in[a, b]\right\} .
$$

Now, we are prepared to present our last assumption.

(iii) The following inequality holds:

$$
\|p\|_{\beta}\left(\max \left\{1,(b-a)^{\beta}\right\}\right)^{2}\left(2 K+k_{\beta}(b-a)\right)<\frac{1}{4},
$$

where $\|p\|_{\beta}$ denotes the norm of the function $p$ in the space $H_{\beta}[a, b]$.

Now, we can formulate our existence result concerning (94).

Theorem 7. Under assumptions (i)-(iii) (94) has at least one solution belonging to the space $H_{\alpha}[a, b]$, where $\alpha$ is arbitrarily fixed number such that $0<\alpha<\beta$.

Proof. At the beginning, let us notice that in view of inclusions (47) we have that if a function $f$ satisfies the Hölder condition with the exponent $\beta$, then it satisfies this condition 
with the exponent $\alpha$. Thus, from assumptions (i) and (ii) it follows that there exists a constant $k_{\alpha}$ such that

$$
\begin{gathered}
|k(t, \tau)-k(s, \tau)| \leqslant k_{\alpha}|t-s|^{\alpha}, \\
|p(t)-p(s)| \leqslant H_{p}^{\alpha}|t-s|^{\alpha}
\end{gathered}
$$

for all $t, s, \tau \in[a, b]$, where the constant $H_{p}^{\alpha}$ associated with the function $p$ is defined by (36).

Now, let us consider the operator $F$ defined on the space $H_{\beta}[a, b]$ by the formula

$$
(F x)(t)=p(t)+x(t) \int_{a}^{b} k(t, \tau) x(\tau) d \tau,
$$

for $t \in[a, b]$.

Further, take an arbitrary function $x \in H_{\beta}[a, b]$. Then, for arbitrarily fixed $t, s \in[a, b]$, in view of assumptions (i) and (ii) we obtain

$$
\begin{aligned}
& \frac{|(F x)(t)-(F x)(s)|}{|t-s|^{\beta}} \\
& \leqslant \frac{|p(t)-p(s)|}{|t-s|^{\beta}} \\
& +\frac{\left|x(t) \int_{a}^{b} k(t, \tau) x(\tau) d \tau-x(s) \int_{a}^{b} k(s, \tau) x(\tau) d \tau\right|}{|t-s|^{\beta}} \\
& \leqslant \\
& \quad H_{p}^{\beta}+\frac{\left|x(t) \int_{a}^{b} k(t, \tau) x(\tau) d \tau-x(s) \int_{a}^{b} k(t, \tau) x(\tau) d \tau\right|}{|t-s|^{\beta}} \\
& +\frac{\left|x(s) \int_{a}^{b} k(t, \tau) x(\tau) d \tau-x(s) \int_{a}^{b} k(s, \tau) x(\tau) d \tau\right|}{|t-s|^{\beta}} \\
& \leqslant \\
& +H_{p}^{\beta}+\frac{|x(t)-x(s)|}{|t-s|^{\beta} \int_{a}^{b}|k(t, \tau)||x(\tau)| d \tau} \\
& +|x(s)| \frac{\int_{a}^{b}|k(t, \tau) x(\tau)-k(s, \tau)||x(\tau)| d \tau}{|t-s|^{\beta}} \\
& \leqslant
\end{aligned}
$$

Hence, in view of the inequalities $\|x\|_{\infty} \leqslant$ $\max \left\{1,(b-a)^{\beta}\right\}\|x\|_{\beta}, H_{x}^{\beta} \leqslant\|x\|_{\beta}$ (cf. (39) and (41)), we derive the following estimate:

$$
\begin{aligned}
\|F x\|_{\beta} \leqslant & |(F x)(a)|+H_{p}^{\beta}+K\|x\|_{\infty} H_{x}^{\beta}+k_{\beta}(b-a)\|x\|_{\infty}^{2} \\
\leqslant & \|p\|_{\beta}+K\|x\|_{\infty}^{2}+K\|x\|_{\infty}\|x\|_{\beta} \\
& +k_{\beta}(b-a)\left(\max \left\{1,(b-a)^{\beta}\right\}\right)^{2}\|x\|_{\beta}^{2} \\
\leqslant & \|p\|_{\beta}+2 K\left(\max \left\{1,(b-a)^{\beta}\right\}\right)^{2}\|x\|_{\beta}^{2} \\
& +k_{\beta}(b-a)\left(\max \left\{1,(b-a)^{\beta}\right\}\right)^{2}\|x\|_{\beta}^{2} \\
\leqslant\|p\|_{\beta}+ & \left(\max \left\{1,(b-a)^{\beta}\right\}\right)^{2}\left(2 K+k_{\beta}(b-a)\right)\|x\|_{\beta}^{2} .
\end{aligned}
$$

This shows that the operator $F$ transforms the space $H_{\beta}[a, b]$ into itself. Moreover, in view of assumption (iii), we infer that $F$ transforms into itself the ball $B_{\beta}$ (in the space $H_{\beta}[a, b]$ ) centered at the zero function $\theta$ and with radius $r_{0}$, where $r_{0}$ is an arbitrary number from the interval $\left[r_{1}, r_{2}\right]$, while

$$
\begin{array}{r}
r_{1}=\frac{1-\sqrt{1-4 \gamma\|p\|_{\beta}}}{2 \gamma}, \\
r_{2}=\frac{1+\sqrt{1-4 \gamma\|p\|_{\beta}}}{2 \gamma},
\end{array}
$$

and $\gamma=\left(\max \left\{1,(b-a)^{\beta}\right\}\right)^{2}\left(2 K+k_{\beta}(b-a)\right)$.

Now, observe that in view of (47) the ball $B_{\beta}$ is contained in the space $H_{\alpha}[a, b]$. Further, keeping in mind the fact established in Example 6, we conclude that the set $B_{\beta}$ is relatively compact in the space $H_{\alpha}[a, b]$. Moreover, taking into account estimate (50), we deduce that $B_{\beta}$ is also closed in the space $H_{\alpha}[a, b]$.

Thus, gathering the above obtained facts, we infer that the set $B_{\beta}$ is a compact and (obviously) convex subset of the space $H_{\alpha}[a, b]$.

In what follows, we will treat the ball $B_{\beta}$ as a subset of the space $H_{\alpha}[a, b]$.

We now show that the operator $F$ is continuous on the space $H_{\alpha}[a, b]$. 
To this end, fix arbitrarily $x \in H_{\alpha}[a, b]$ and a number $\delta>$ 0 . Assume that $y \in H_{\alpha}[a, b]$ is an arbitrary function such that $\|x-y\|_{\alpha} \leqslant \delta$. Then, for arbitrary $t, s \in[a, b]$ we obtain

$$
\begin{aligned}
& \frac{|[(F x)(t)-(F y)(t)]-[(F x)(s)-(F y)(s)]|}{|t-s|^{\alpha}} \\
& =\mid \frac{\left[x(t) \int_{a}^{b} k(t, \tau) x(\tau) d \tau-y(t) \int_{a}^{b} k(t, \tau) y(\tau) d \tau\right]}{|t-s|^{\alpha}} \\
& -\frac{\left[x(s) \int_{a}^{b} k(s, \tau) x(\tau) d \tau-y(s) \int_{a}^{b} k(s, \tau) y(\tau) d \tau\right]}{|t-s|^{\alpha}} \mid \\
& \leqslant \mid \frac{\left[x(t) \int_{a}^{b} k(t, \tau) x(\tau) d \tau-y(t) \int_{a}^{b} k(t, \tau) x(\tau) d \tau\right]}{|t-s|^{\alpha}} \\
& +\frac{\left[y(t) \int_{a}^{b} k(t, \tau) x(\tau) d \tau-y(t) \int_{a}^{b} k(t, \tau) y(\tau) d \tau\right]}{|t-s|^{\alpha}} \\
& -\frac{\left[x(s) \int_{a}^{b} k(s, \tau) x(\tau) d \tau-y(s) \int_{a}^{b} k(s, \tau) x(\tau) d \tau\right]}{|t-s|^{\alpha}} \\
& -\frac{\left[y(s) \int_{a}^{b} k(s, \tau) x(\tau) d \tau-y(s) \int_{a}^{b} k(s, \tau) y(\tau) d \tau\right]}{|t-s|^{\alpha}} \mid \\
& =\mid\left([x(t)-y(t)] \int_{a}^{b} k(t, \tau) x(\tau) d \tau\right. \\
& \left.+y(t) \int_{a}^{b} k(t, \tau)[x(\tau)-y(\tau)] d \tau\right)\left(|t-s|^{\alpha}\right)^{-1} \\
& -\left([x(s)-y(s)] \int_{a}^{b} k(s, \tau) x(\tau) d \tau\right. \\
& \left.+y(s) \int_{a}^{b} k(s, \tau)[x(\tau)-y(\tau)] d \tau\right)\left(|t-s|^{\alpha}\right)^{-1} \mid \\
& \leqslant \frac{|[x(t)-y(t)]-[x(s)-y(s)]|\left|\int_{a}^{b} k(t, \tau) x(\tau) d \tau\right|}{|t-s|^{\alpha}} \\
& +\frac{|x(s)-y(s)|\left|\int_{a}^{b}[k(t, \tau)-k(s, \tau)] x(\tau) d \tau\right|}{|t-s|^{\alpha}} \\
& +\mid\left(y(t) \int_{a}^{b} k(t, \tau)[x(\tau)-y(\tau)] d \tau\right. \\
& \left.-y(s) \int_{a}^{b} k(s, \tau)[x(\tau)-y(\tau)] d \tau\right) \mid\left(|t-s|^{\alpha}\right)^{-1}
\end{aligned}
$$$$
\leqslant \frac{|[x(t)-y(t)]-[x(s)-y(s)]|}{|t-s|^{\alpha}}\|x\|_{\infty} \int_{a}^{b}|k(t, \tau)| d \tau
$$$$
+\{|[x(s)-y(s)]-[x(a)-y(a)]|
$$$$
+|x(a)-y(a)|\}\|x\|_{\infty} \frac{\int_{a}^{b}|k(t, \tau)-k(s, \tau)| d \tau}{|t-s|^{\alpha}}
$$$$
+\mid\left(y(t) \int_{a}^{b} k(t, \tau)[x(\tau)-y(\tau)] d \tau\right.
$$$$
\left.-y(s) \int_{a}^{b} k(t, \tau)[x(\tau)-y(\tau)] d \tau\right) \mid\left(|t-s|^{\alpha}\right)^{-1}
$$$$
+\mid\left(y(s) \int_{a}^{b} k(t, \tau)[x(\tau)-y(\tau)] d \tau\right.
$$$$
\left.-y(s) \int_{a}^{b} k(s, \tau)[x(\tau)-y(\tau)] d \tau\right) \mid\left(|t-s|^{\alpha}\right)^{-1}
$$$$
\leqslant\|x-y\|_{\alpha}\|x\|_{\infty} \sup \left\{\int_{a}^{b}|k(t, \tau)| d \tau: t \in[a, b]\right\}
$$$$
+\sup _{t, s \in[a, b]}\{|[x(t)-y(t)]-[x(s)-y(s)]|\}
$$$$
\times\|x\|_{\infty} \int_{a}^{b} \frac{k_{\beta}|t-s|^{\beta}}{|t-s|^{\alpha}} d \tau
$$$$
+|x(a)-y(a)|\|x\|_{\infty} \frac{\int_{a}^{b} k_{\beta}|t-s|^{\beta}}{|t-s|^{\alpha}} d \tau
$$$$
+\frac{|y(t)-y(s)|}{|t-s|^{\alpha}} \int_{a}^{b}|k(t, \tau)||x(\tau)-y(\tau)| d \tau
$$$$
+|y(s)| \int_{a}^{b} \frac{|k(t, \tau)-k(s, \tau)|}{|t-s|^{\alpha}}|x(\tau)-y(\tau)| d \tau
$$$$
\leqslant K\|x\|_{\infty}\|x-y\|_{\alpha}+k_{\beta}(b-a)^{\beta-\alpha+1}\|x\|_{\infty}
$$$$
\times \sup \left\{\frac{|[x(t)-y(t)]-[x(s)-y(s)]|}{|t-s|^{\alpha}}\right.
$$$$
\left.\times|t-s|^{\alpha}: t, s \in[a, b], t \neq s\right\}
$$$$
+|x(a)-y(a)|\|x\|_{\infty} k_{\beta}(b-a)^{\beta-\alpha+1}
$$$$
+\frac{H_{y}^{\alpha}|t-s|^{\alpha}}{|t-s|^{\alpha}} \int_{a}^{b}|k(t, \tau)|
$$$$
\times\left\{\sup _{t \in[a, b]}\{|[x(t)-y(t)]-[x(a)-y(a)]|\right.
$$$$
+|[x(a)-y(a)]|\}\} d \tau
$$

$+\|y\|_{\infty}$ 


$$
\begin{aligned}
& \times \int_{a}^{b} \frac{k_{\beta}|t-s|^{\beta}}{|t-s|^{\alpha}}\left\{\sup _{t \in[a, b]}\{|[x(t)-y(t)]-[x(a)-y(a)]|\right. \\
& +|[x(a)-y(a)]|\}\} d \tau \\
& \leqslant K\|x\|_{\infty}\|x-y\|_{\alpha} \\
& +k_{\beta}(b-a)^{\beta-\alpha+1}\|x\|_{\infty} \\
& \times \sup \left\{\frac{|[x(t)-y(t)]-[x(s)-y(s)]|}{|t-s|^{\alpha}}\right. \\
& : t, s \in[a, b], t \neq s\}(b-a)^{\alpha} \\
& +k_{\beta}(b-a)^{\beta-\alpha+1}\|x\|_{\infty}|x(a)-y(a)| \\
& +H_{y}^{\alpha} \sup \{|[x(t)-y(t)]-[x(s)-y(s)]| \\
& : t, s \in[a, b]\} \int_{a}^{b}|k(t, \tau)| d \tau \\
& +H_{y}^{\alpha}|x(a)-y(a)| \int_{a}^{b}|k(t, \tau)| d \tau \\
& +k_{\beta}\|y\|_{\infty}(b-a)^{\beta-\alpha+1} \\
& \times \sup \{|[x(t)-y(t)]-[x(s)-y(s)]| \\
& : t, s \in[a, b]\} \\
& +k_{\beta}(b-a)^{\beta-\alpha+1}\|y\|_{\infty}|x(a)-y(a)| \\
& \leqslant K\|x\|_{\infty}\|x-y\|_{\alpha}+k_{\beta}(b-a)^{\beta+1}\|x\|_{\infty}\|x-y\|_{\alpha} \\
& +k_{\beta}(b-a)^{\beta-\alpha+1}\|x\|_{\infty}|x(a)-y(a)| \\
& +H_{y}^{\alpha} K \sup \left\{\frac{|[x(t)-y(t)]-[x(s)-y(s)]|}{|t-s|^{\alpha}}\right. \\
& : t, s \in[a, b], t \neq s\}(b-a)^{\alpha} \\
& +H_{y}^{\alpha} K|x(a)-y(a)| \\
& +k_{\beta}\|y\|_{\infty}(b-a)^{\beta-\alpha+1} \\
& \times \sup \left\{\frac{|[x(t)-y(t)]-[x(s)-y(s)]|}{|t-s|^{\alpha}}\right. \\
& : t, s \in[a, b], t \neq s\}(b-a)^{\alpha} \\
& +k_{\beta}(b-a)^{\beta-\alpha+1}\|y\|_{\infty}|x(a)-y(a)| \\
& \leqslant K\|x\|_{\infty}\|x-y\|_{\alpha}+k_{\beta}(b-a)^{\beta+1}\|x\|_{\infty}\|x-y\|_{\alpha} \\
& +k_{\beta}(b-a)^{\beta-\alpha+1}\|x\|_{\infty}|x(a)-y(a)|
\end{aligned}
$$$$
+H_{y}^{\alpha} K(b-a)^{\alpha}\|x-y\|_{\alpha}+H_{y}^{\alpha} K|x(a)-y(a)|
$$$$
+k_{\beta}\|y\|_{\infty}(b-a)^{\beta+1}\|x-y\|_{\alpha}
$$$$
+k_{\beta}(b-a)^{\beta-\alpha+1}\|y\|_{\infty}|x(a)-y(a)| .
$$

Further, let us observe that we have the following estimates:

$$
\begin{aligned}
|(F x)(a)-(F y)(a)| & \mid p(a)+x(a) \int_{a}^{b} k(a, \tau) x(\tau) d \tau \\
= & \left|p(a)-y(a) \int_{a}^{b} k(a, \tau) y(\tau) d \tau\right| \\
= & \mid x(a) \int_{a}^{b} k(a, \tau) x(\tau) d \tau-x(a) \int_{a}^{b} k(a, \tau) y(\tau) d \tau \\
& +x(a) \int_{a}^{b} k(a, \tau) y(\tau) d \tau \\
& -y(a) \int_{a}^{b} k(a, \tau) y(\tau) d \tau \mid \\
\leqslant & K\left(\|x\|_{\infty}\|x-y\|_{\infty}+\|x-y\|_{\alpha}\|y\|_{\infty}\right) . \\
& +\left|x(a) \int_{a}^{b} k(a, \tau)(x(\tau)-y(\tau)) d \tau\right| \\
\leqslant & |x(a)| \int_{a}^{b}|k(a, \tau)||x(\tau)-y(\tau)| d \tau \\
& +\left|(x(a)-y(a)) \int_{a}^{b} k(a, \tau) y(\tau) d \tau\right|
\end{aligned}
$$


Combining the above established facts and (41), in view of (103) and (104), we obtain following estimates:

$$
\begin{aligned}
\|f x-f y\|_{\alpha} & \leqslant K\left(\max \left\{1,(b-a)^{\alpha}\right\}\right)^{2}\left(2\|x\|_{\alpha}+\delta\right) \delta \\
& +\left(K+k_{\beta}(b-a)^{\beta+1}\right)\|x\|_{\infty} \delta \\
& +k_{\beta}(b-a)^{\beta-\alpha+1}\|x\|_{\infty} \delta+K(b-a)^{\alpha}\left(\|x\|_{\alpha}+\delta\right) \delta \\
& +K\left(\|x\|_{\alpha}+\delta\right) \delta \\
& +k_{\beta}(b-a)^{\beta+1} \max \left\{1,(b-a)^{\alpha}\right\}\left(\|x\|_{\alpha}+\delta\right) \delta \\
& +k_{\beta}(b-a)^{\beta-\alpha+1} \max \left\{1,(b-a)^{\alpha}\right\}\left(\|x\|_{\alpha}+\delta\right) \delta .
\end{aligned}
$$

This shows that the operator $F$ is continuous at the point $x \in H_{\alpha}[a, b]$. Since $x$ was chosen arbitrarily, we deduce that $F$ is continuous on the Hölder space $H_{\alpha}[a, b]$. Particularly, the operator $F$ maps continuously the ball $B_{\beta}$ into itself. Thus, taking into account the fact that the set $B_{\beta}$ is compact in the space $H_{\alpha}[a, b]$ and applying the classical Schauder fixed point principle, we complete the proof. ple.

Now, we illustrate the above obtained result by an exam-

Example 8. Let us consider the following quadratic integral equation:

$$
x(t)=\sqrt{q t+r}+x(t) \int_{0}^{1} \sqrt[3]{m t^{2}+\tau} x(\tau) d \tau
$$

where $t \in[0,1]$ and $q, r, m$ are positive constants.

Observe that (107) is a particular case of (94) if we put $p(t)=\sqrt{q t+r}, k(t, \tau)=\sqrt[3]{m t^{2}+\tau}$. Further, we get

$$
|p(t)-p(s)| \leqslant \sqrt{q}|t-s|^{1 / 2} .
$$

Moreover, using the inequality proved in [12], we obtain

$$
|k(t, \tau)-k(s, \tau)|=\left|\sqrt[3]{m t^{2}+\tau}-\sqrt[3]{m s^{2}+\tau}\right| \leqslant \sqrt[3]{m}|t-s|^{2 / 3} .
$$

Thus, in our considerations related to Theorem 7 we can take $\beta=1 / 2$. Then obviously we have

$$
\begin{aligned}
|k(t, \tau)-k(s, \tau)| \leqslant \sqrt[3]{m}|t-s|^{2 / 3} & =\sqrt[3]{m}|t-s|^{1 / 2}|t-s|^{1 / 6} \\
& \leqslant \sqrt[3]{m}|t-s|^{1 / 2}
\end{aligned}
$$

Hence we infer that $k_{\beta}=k_{1 / 2}=\sqrt[3]{m}$. Apart from this, we get

$$
\begin{aligned}
\|p\|_{1 / 2} & =|p(0)|+\sup \left\{\frac{|p(t)-p(s)|}{|t-s|^{1 / 2}}: t, s \in[0,1], t \neq s\right\} \\
& =\sqrt{r}+\sqrt{q} .
\end{aligned}
$$

This shows that functions $p(t)$ and $k(t, \tau)$ involved in (107) satisfy assumptions (i) and (ii) of Theorem 7.

Further, we can calculate that

$$
\begin{aligned}
K & =\sup \left\{\int_{0}^{1}|k(t, \tau)| d \tau: t \in[0,1]\right\} \\
& =\sup \left\{\int_{0}^{1} \sqrt[3]{m t^{2}+\tau} d \tau: t \in[0,1]\right\} \\
& =\sup \left\{\frac{3}{4}\left[\sqrt[3]{\left(m t^{2}+1\right)^{4}}-\sqrt[3]{\left(m t^{2}\right)^{4}}\right]: t \in[0,1]\right\} \\
& =\frac{3}{4}\left[\sqrt[3]{(m+1)^{4}}-\sqrt[3]{m^{4}}\right] .
\end{aligned}
$$

Hence we deduce that the inequality from assumption (iii) is satisfied provided

$$
(\sqrt{q}+\sqrt{r})\left\{\sqrt[3]{m}+\frac{3}{2}\left[\sqrt[3]{(m+1)^{4}}-\sqrt[3]{m^{4}}\right]\right\}<\frac{1}{4}
$$

It is easy to check that the above inequality is satisfied if we put, for example, $q=r=1 / 216, m=1 / 128$.

Finally, applying Theorem 7 , we conclude that (107) has at least one solution in the space $H_{\alpha}[0,1]$ with $0<\alpha<1 / 2$, provided inequality (113) is satisfied.

\section{References}

[1] N. Danford and J. T. Schwartz, Linear Operators I, International Publishing, Leyden, The Netherlands, 1963.

[2] A. N. Kolmogorov and S. V. Fomīn, Introductory Real Analysis, Dover Publications, New York, NY, USA, 1975.

[3] W. Rudin, Functional Analysis, McGraw-Hill, New York, NY, USA, 2nd edition, 1991.

[4] J. Wloka, Funktionalanalysis und Anwendungen, Walter de Gruyter, Berlin, Germany, 1971.

[5] J. Appell and P. P. Zabrejko, Nonlinear Superposition Operators, vol. 95 of Cambridge Tracts in Mathematics, Cambridge University Press, Cambridge, UK, 1990.

[6] J. Matkowski, "Functional equations and Nemytskil operators," Funkcialaj Ekvaciog, vol. 25, no. 2, pp. 127-132, 1982.

[7] I. K. Argyros, "On a class of quadratic integral equations with perturbation," Functiones et Approximatio Commentarii Mathematici, vol. 20, pp. 51-63, 1992.

[8] L. W. Busbridge, The Mathematics of Radiative Transfer, Cambridge University Press, Cambridge, UK, 1960.

[9] K. M. Case and P. F. Zweifel, Linear Transport Theory, AddisonWesley, Reading, Mass, USA, 1967.

[10] S. Chandrasekhar, Radiative Transfer, Oxford University Press, London, UK, 1950.

[11] S. Hu, M. Khavanin, and W. Zhuang, "Integral equations arising in the kinetic theory of gases," Applicable Analysis, vol. 34, no. 3-4, pp. 261-266, 1989.

[12] R. P. Agarwal, J. Banaś, K. Banaś, and D. O’Regan, “Solvability of a quadratic Hammerstein integral equation in the class of functions having limits at infinity," Journal of Integral Equations and Applications, vol. 23, no. 2, pp. 157-181, 2011. 


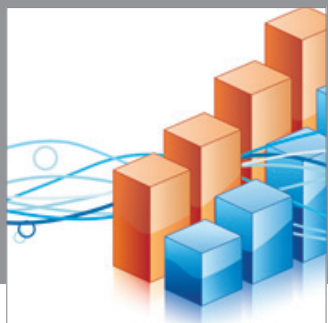

Advances in

Operations Research

mansans

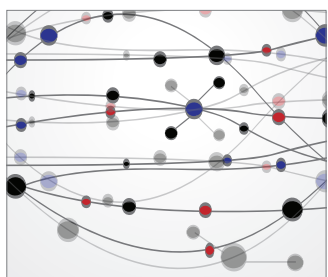

The Scientific World Journal
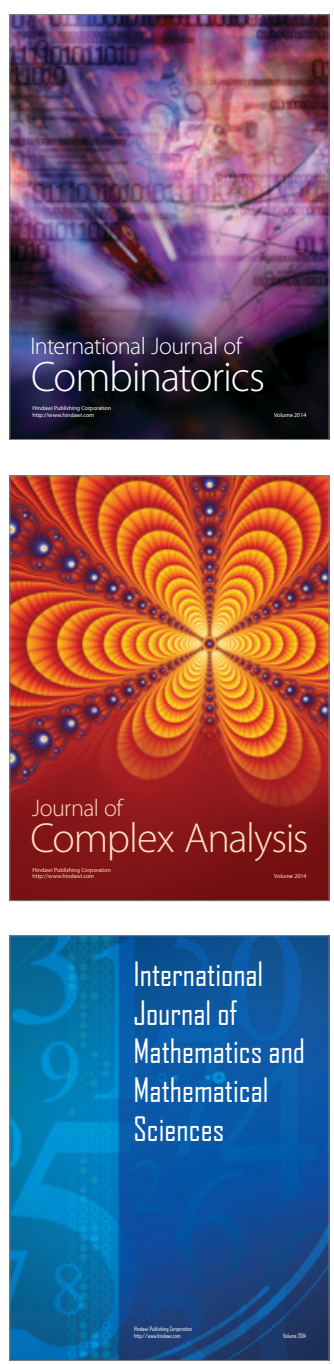
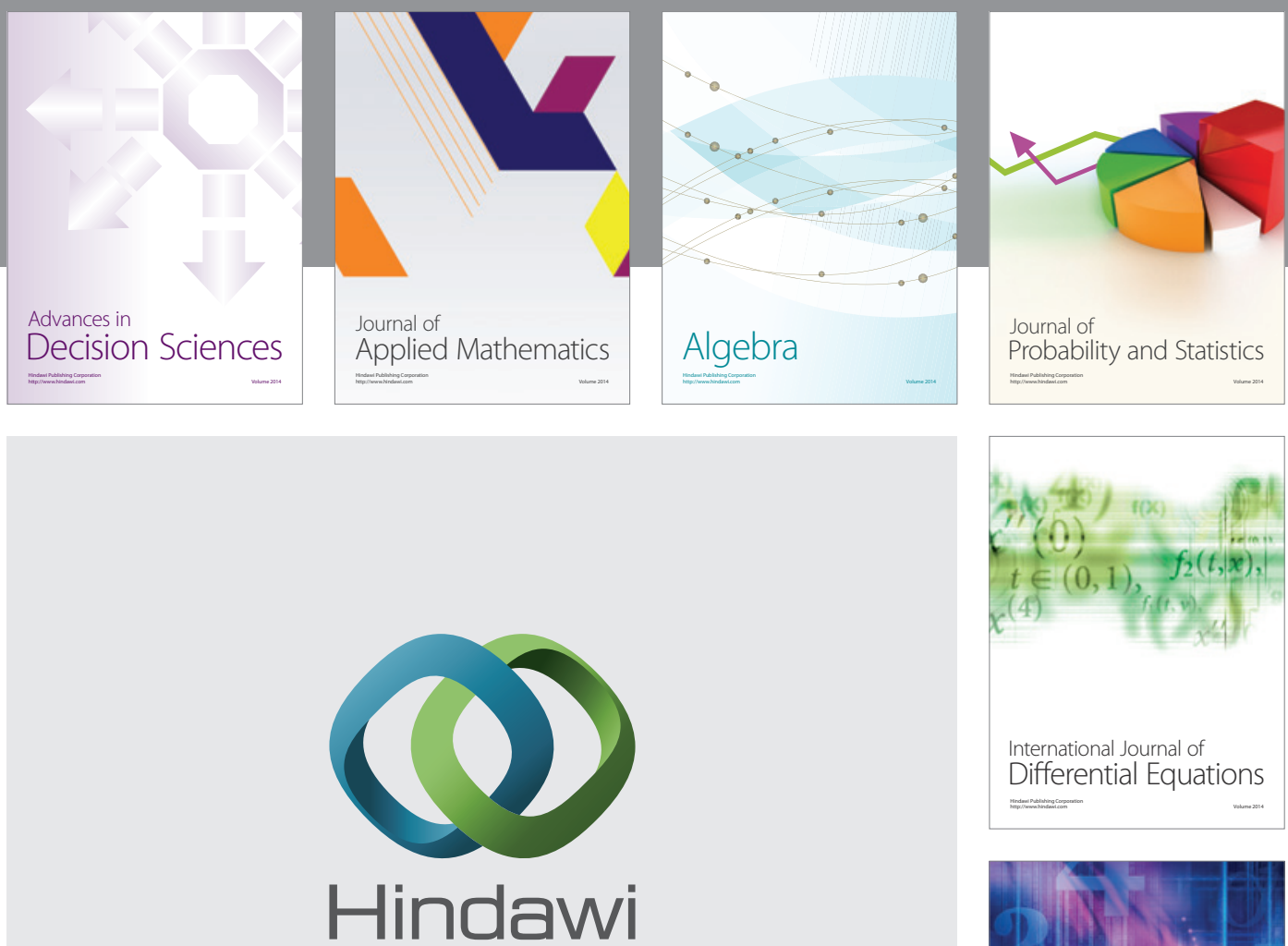

Submit your manuscripts at http://www.hindawi.com
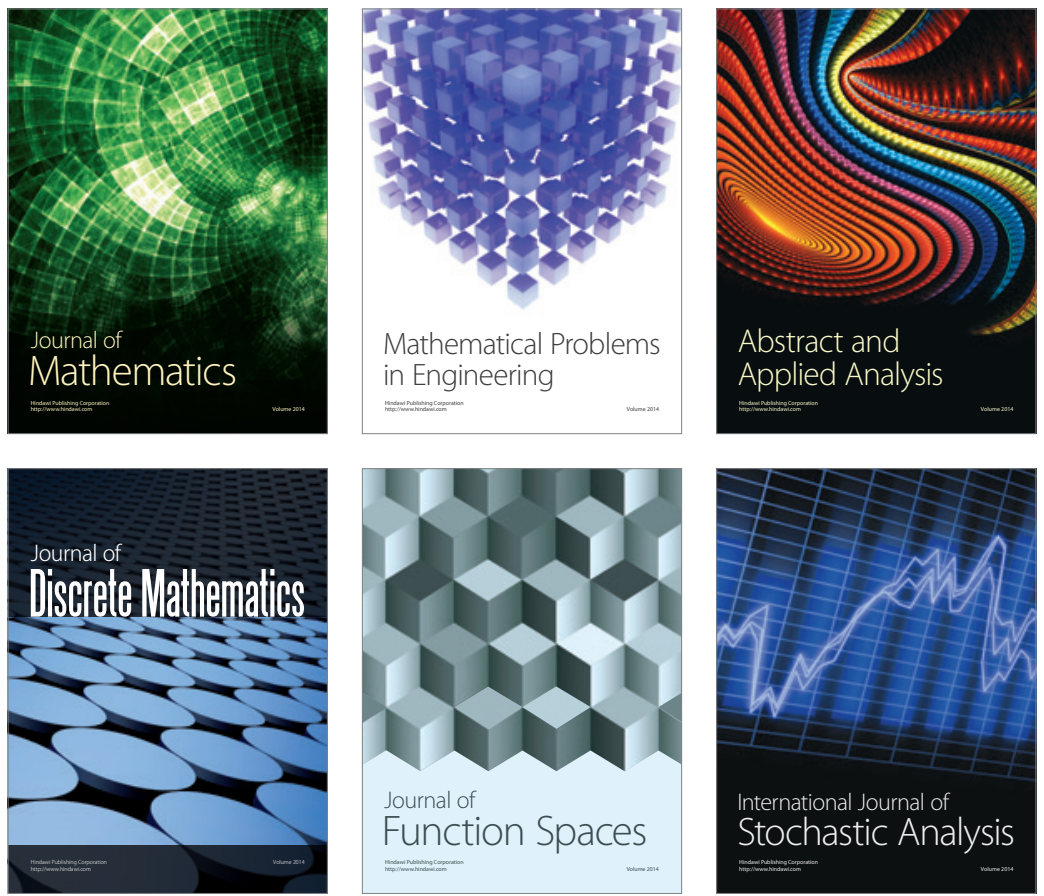

Journal of

Function Spaces

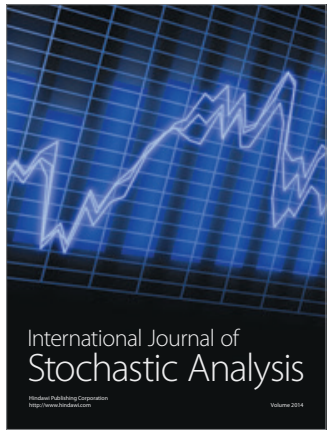

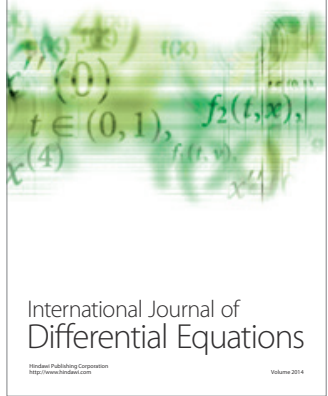
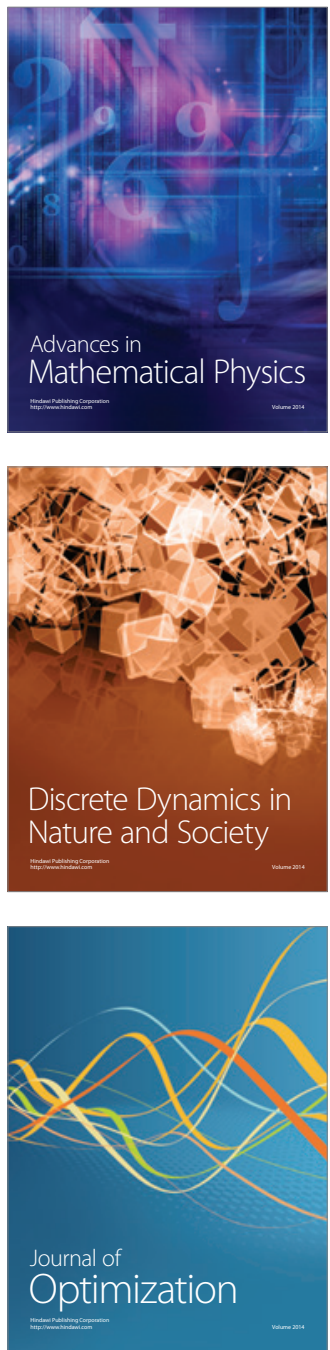